\title{
Neurofaciodigitorenal syndrome
}

INSERM

\section{Source}

INSERM. (1999). Orphanet: an online rare disease and orphan drug data base.

Neurofaciodigitorenal syndrome. ORPHA:2673

Neurofaciodigitorenal syndrome is a rare multiple developmental anomalies syndrome characterized by neurological abnormalities (including megalencephaly, hypotonia, intellectual disability, abnormal EEG), dysmorphic facial features (high prominent forehead, grooved nasal tip, ptosis, ear anomalies) and acrorenal defects (such as triphalang ism, broad halluces, unilateral renal agenesis). Additionally, intrauterine growth restriction, short stature and congenital heart defects may be associated. There have been no further descriptions in the literature since 1997. 